\title{
"An analysis of how Fortune 500 companies respond to users replying to company tweets"
}

\begin{tabular}{|c|c|}
\hline AUTHORS & $\begin{array}{l}\text { Michael North (D https://orcid.org/0000-0001-7362-4058 } \\
\text { Cong Li } \\
\text { Jiangmeng Liu }\end{array}$ \\
\hline ARTICLE INFO & $\begin{array}{l}\text { Michael North, Cong Li and Jiangmeng Liu (2017). An analysis of how Fortune } \\
500 \text { companies respond to users replying to company tweets. Innovative } \\
\text { Marketing, 13(4), 17-24. doi:10.21511/im.13(4).2017.02 }\end{array}$ \\
\hline DOI & http://dx.doi.org/10.21511/im.13(4).2017.02 \\
\hline RELEASED ON & Thursday, 07 December 2017 \\
\hline RECEIVED ON & Wednesday, 01 November 2017 \\
\hline ACCEPTED ON & Friday, 01 December 2017 \\
\hline LICENSE & $\begin{array}{l}(c) \text { EY } \\
\text { This work is licensed under a Creative Commons Attribution } 4.0 \text { International } \\
\text { License }\end{array}$ \\
\hline JOURNAL & "Innovative Marketing " \\
\hline ISSN PRINT & $1814-2427$ \\
\hline ISSN ONLINE & $1816-6326$ \\
\hline PUBLISHER & LLC "Consulting Publishing Company "Business Perspectives" \\
\hline FOUNDER & LLC "Consulting Publishing Company "Business Perspectives" \\
\hline
\end{tabular}

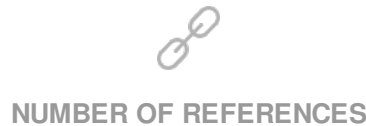

41
NUMBER OF FIGURES

1
NUMBER OF TABLES

1

(C) The author(s) 2021. This publication is an open access article. 
Michael North (USA), Cong Li (USA), Jiangmeng Liu (USA)

\title{
An analysis of how Fortune 500 companies respond to users replying to company tweets
}

\begin{abstract}
With hundreds of millions of active users generating almost a half of a billion tweets each day, Twitter has solidified itself as one of the most popular websites in today's digital world. Because of this popularity, companies seeking to leverage the large audience have gravitated toward Twitter. This study examines how the Fortune 500 uses Twitter by analyzing 9,122 corporate tweets and 1,509 user replies through the use of content analysis. Examined factors include interactivity (non-interactive vs. reactive vs. interactive), company type (B2B vs. B2C), and user reply valence (positive vs. neutral vs. negative). Company response time to user replies is also investigated. The study results point to interactive tweets generating the most engagement. B2Cs not only respond faster to user replies but also generate more engagement than B2Bs. Negative replies can decrease engagement for B2Bs and B2Cs, but the influence on B2Bs is more profound. Companies responded the fastest to negative replies followed by positive replies and neutral replies, respectively. Thus, a company should assess its own business practices, target audience, and ability to perform customer service before creating a social media account such as Twitter.
\end{abstract}

Keywords: Twitter, interactivity, B2B, B2C, reply, retweet, like, Fortune 500, response time.

JEL Classification: M30, M31, M39.

Received on: $1^{\text {st }}$ of November, 2017.

Accepted on: $1^{\text {st }}$ of December, 2017.

\section{Introduction}

Social media platforms such as Facebook and Twitter are important strategic communication vehicles for companies to build relationships and initiate conversations with the public. As argued by Rim and Song (2016), organizations are now facing more challenges regarding interactions with the public on social media, especially when responding to negative user comments, and thus, effective social media communication strategies need to be identified. The current research contributes to this body of literature by analyzing 9,122 corporate tweets and 1,509 user replies collected from Twitter, a popular social media platform "used by virtually all big Western companies either for marketing or to respond to customers - and sometimes both" (Parmar, 2015). The interaction between company and user needs to be examined as more companies gravitate toward Twitter to meet the ever increasing number of users. Specifically, this study examines the Twitter practices of the largest and most successful companies in the United States (the Fortune 500). The study results will give a sense of how interactive companies are on Twitter along with how the tone of a user's reply can affect company response time along with future engagement among users.

(C) Limited Liability Company "Consulting Publishing Company "Business Perspectives", 2017.

Michael North, Ph.D., Assistant Professor, Communication Department, Central Connecticut State University, Connecticut, USA.

Cong Li, Ph.D., Associate Professor, School of Communication, University of Miami, Coral Gables, Florida, USA

Jiangmeng Liu, Ph.D., Assistant Professor, Communication Department, Seattle University, Seattle, Washington, USA.

\section{Literature review}

1.1. Should companies respond to individual users on Twitter? Although many companies have a poor record for engaging with individual social media users (Waters \& Williams, 2011), companies should respond to user comments from an interactivity standpoint. According to the classic contingency viewpoint of interactivity, there are three levels of interactivity: non-interactive, reactive, and interactive (Rafaeli, 1988; Sundar, Brwon, \& Kalyanaraman, 2003). Non-interactive communication is the lowest level of interactivity, meaning that the messages exchanged between two parties are not contingent on each other. However, if the latter message refers to the previous message, the communication is reactive. Furthermore, if the latter message refers to not only the previous message, but also the previous message exchange, such as A replies to B's reply on A's first message, it is a fully interactive communication. The level of reciprocity of message exchange will influence the perceived quality of communication (Rafaeli, 1988). With an increasing level of interactivity, people will feel they are receiving better quality information, which also increases perceived control, sense of self-efficacy, and attitude toward the medium, as well as the owner of the medium (Lee \& Park, 2013; Song \& Zinkhan, 2008; Sundar et al., 2003).

This study is focused on how the Fortune 500 companies interact with users on Twitter. In such a context, a company's following is an online community and messages from the company that demonstrate contingent interactivity lead to 
increased potential for user participation (Wise, Hamman, \& Thorson, 2006). The effort to be more responsive is expected to increase positivity toward the company (Saffer, Sommerfeldt, \& Taylor, 2013). To examine its effect, this study operationalizes interactivity at three levels: 1) non-interactive (i.e., a company posts a tweet but no user replies to the tweet); 2) reactive (i.e., a company posts a tweet and a user replies to the tweet); and 3) interactive (i.e., a company posts a tweet, a user replies to the tweet, and the company replies to the user's reply). It is anticipated that a higher level of interactivity will generate more user engagement in the form of likes, retweets, and replies in a Twitter context:

H1: The higher the level of interactivity, the more likes, retweets, and replies a company tweet is expected to receive.

\subsection{What kind of user replies should companies} respond to? Message valence plays an important role on social media. Too much positivity has the potential to be perceived as overt advertising and manipulative (Roering \& Paul, 1976). Negative information establishes credibility and humanizes the message, but if too many drawbacks are presented, consumers will choose an alternative (Eisend, 2013). Users tend to emphasize negative comments because positive comments reflect the user while negative comments focus on the product itself (Chen \& Lurie, 2013). In line with negativity bias that emphasizes the downside over the upside, "negative information is most likely to be paid attention to, remembered, and then used for judgment" (Martin, 2008, p. 184).

Since consumers place a fair amount of importance on negativity concerning products or brands, the ability of a negative user reply in generating other users' replies, retweets, and likes deserves further examination. The following research question is proposed for this purpose:

RQ: How does a user's negative reply influence other users' replies, retweets, and likes directed at the company?

\subsection{How quickly should companies respond to} users? Admitting to faults and even championing adverse situations is a tactic used by strategic communication practitioners to demonstrate transparency. To avoid the appearance of impropriety, companies should be as transparent as possible, especially when using social media because failing to do so can lead to diminished reputation (Sweetser, 2010). A simple way to appear transparent is to respond quickly to criticism or negative situations raised by users. Short response times convey respect while lengthy response times are likely to elicit negative reactions (Kalman et al., 2006). The confusion of a long pause or a permanent silence can be maddening for a user and debilitating for a company (Kalman \& Rafaeli, 2011). Time is an important concept when examining Twitter. Users appreciate consistent updates and are more easily persuaded when expectations are met in terms of response time (Westerman, Spence, \& Heide, 2014). Because rapid response times are appreciated by users, companies should seek to respond quickly to negativity to diffuse the situation. Additionally, business-to-consumer companies (B2Cs) - those experienced in interacting with individual consumers - are expected to respond quickly to users' messages as opposed to business-to-business companies (B2Bs) that are perhaps more accustomed to traditional strategic communication or less equipped to monitor social media. Based on this rationale, the following hypotheses are proposed:

H2: A company will respond fastest when the valence of a user reply is negative as opposed to positive or neutral.

H3: On average, a B2C company will respond faster to a user reply than a B2B company.

H4: There will be an interaction effect between user reply valence and company type on response time such that a negative user reply will generate a faster response from a $\mathrm{B} 2 \mathrm{C}$ company than a $\mathrm{B} 2 \mathrm{~B}$ company but there will not be such a difference when the reply is positive or neutral.

\section{Method}

Tweets were collected from companies tweeting naturally over a five-year period and content analyzed for various characteristics outlined within this section. First, the Twitter accounts were located. The most accurate way to do this is to visit the corporate website and then click on the Twitter logo to access the corporate Twitter page. Most of the companies had the link to its Twitter page displayed on either the Home screen or within a Social Media, Media Relations, or Contact Us page. If there was not a link to a Twitter page on the corporate website, a simple Google search with the company name and the word "Twitter" would usually present the link to the corporate Twitter page as the first result. A total of 420 companies were found to have a Twitter account. One tweet per month was collected for each of these companies from January 2009 to December 2013. A random number was 
selected that served as the date for the specific month. If a company posted multiple tweets on the selected date, these multiple tweets were each assigned a number and one was randomly selected. If there were no tweets on the selected date, the tweet posted on the next closest day in the specific month was used. Once a tweet was selected, a screenshot of the tweet was saved along with a screenshot of the two most recent user replies to that tweet. This sampling procedure yielded 9,122 tweets and 1,509 user replies in this study.

To analyze these tweets and replies, the following procedure was followed. First, the number of each tweet's replies, retweets, and likes was recorded. Second, each user reply was coded for its purpose: 1) compliment, 2) complaint, 3) question, 4) user's self-promotion, and 5) give feedback/opinion. The data from this procedure were used for both the interactivity and reply valence portions of this study. For interactivity, this study explored instances in the sample when a company tweeted with no user reply (non-interactive); when a company tweeted and a user replied (reactive); and when a company tweeted, a user replied, and the company replied to the user reply (interactive). As for valence, user replies were classified as positive, neutral, or negative. Compliments were considered as positive; questions, self-promotion, and feedback were regarded as neutral; and complaints were negative.

In a pretest, two coders independently coded the tweets of five randomly selected companies and their coding appeared to be reliable (overall agreement $=92.8 \%$ ). The discrepancies were resolved by a later discussion. The remaining sample tweets were evenly divided and coded by each coder.

This study also examines B2B versus B2C tweeting behavior. The procedure for this involved capturing the text from the "About" section on corporate websites, removing any identifying text, and submitting the text to two coders. The coders were instructed to look for text that explains selling products or services to other businesses or selling products and services to consumers. Using this procedure, the overall reliability was $96.8 \%$ in terms of percent agreement. Once reliability was established, the coders reconciled differences, split the rest of the sample, and coded independently.

The last focus of this study is the response time. To collect this information, the time stamp for the users' replies and the companies' responses were recorded and computed in minutes.

\section{Results}

3.1. Descriptive statistics. Among the 9,122 tweets, $12.2 \%$ received replies from users. Most of the replies were neutral $(54.3 \%)$. The remainder of replies was coded as positive $(31.2 \%)$ or negative $(14.5 \%)$.

3.2. Tests of hypotheses. To test the proposed hypotheses, a 3 (non-interactive vs. reactive vs. interactive) $\times 2$ (B2B vs. B2C) MANOVA test was first conducted, with the number of replies, retweets, and likes as dependent variables. The results revealed that both main effects of interactivity (Pillai's trace $=.217, F(6,18,230)=370.62, p<$ $.001)$ and company type (Pillai's trace $=.033, F(3$, $9,114)=102.64, p<.001)$ and interaction effect (Pillai's trace $=.042, F(6,18,230)=64.95, p<$ $.001)$ were statistically significant.

Following ANOVA tests showed that a tweet from B2C companies $\left(M_{\text {replies }}=.78, M_{\text {retweets }}=14.27, M_{\text {likes }}\right.$ $=7.52$ ) received significantly more replies, retweets, and likes (all $p$ s $<.001$ ) than B2B companies $\left(M_{\text {replies }}=.13, M_{\text {retweets }}=2.46, M_{\text {likes }}=.67\right)$. It was also found that the level of interactivity was significant for all three dependent variables (all $p s<$ $.001)$. Post hoc tests indicated that non-interactive tweets $\left(M_{\text {replies }}=.004, M_{\text {retweets }}=2.83, M_{\text {likes }}=\right.$ 1.00) received significantly fewer replies, retweets, and likes than both reactive $\left(M_{\text {replies }}=\right.$ 2.86, $\left.M_{\text {retweets }}=38.78, \quad M_{\text {likes }}=23.75\right)$ and interactive tweets $\left(M_{\text {replies }}=6.59, M_{\text {retweets }}=74.62\right.$, $\left.M_{\text {likes }}=28.15\right)$. Reactive tweets also received significantly fewer replies and retweets than interactive tweets. Thus, H1 was supported. Furthermore, the ANOVA tests indicated significant interaction effects on all three dependent variables (all $p$ s $<.001$ ). The directions of these effects are shown in Figure 1. 

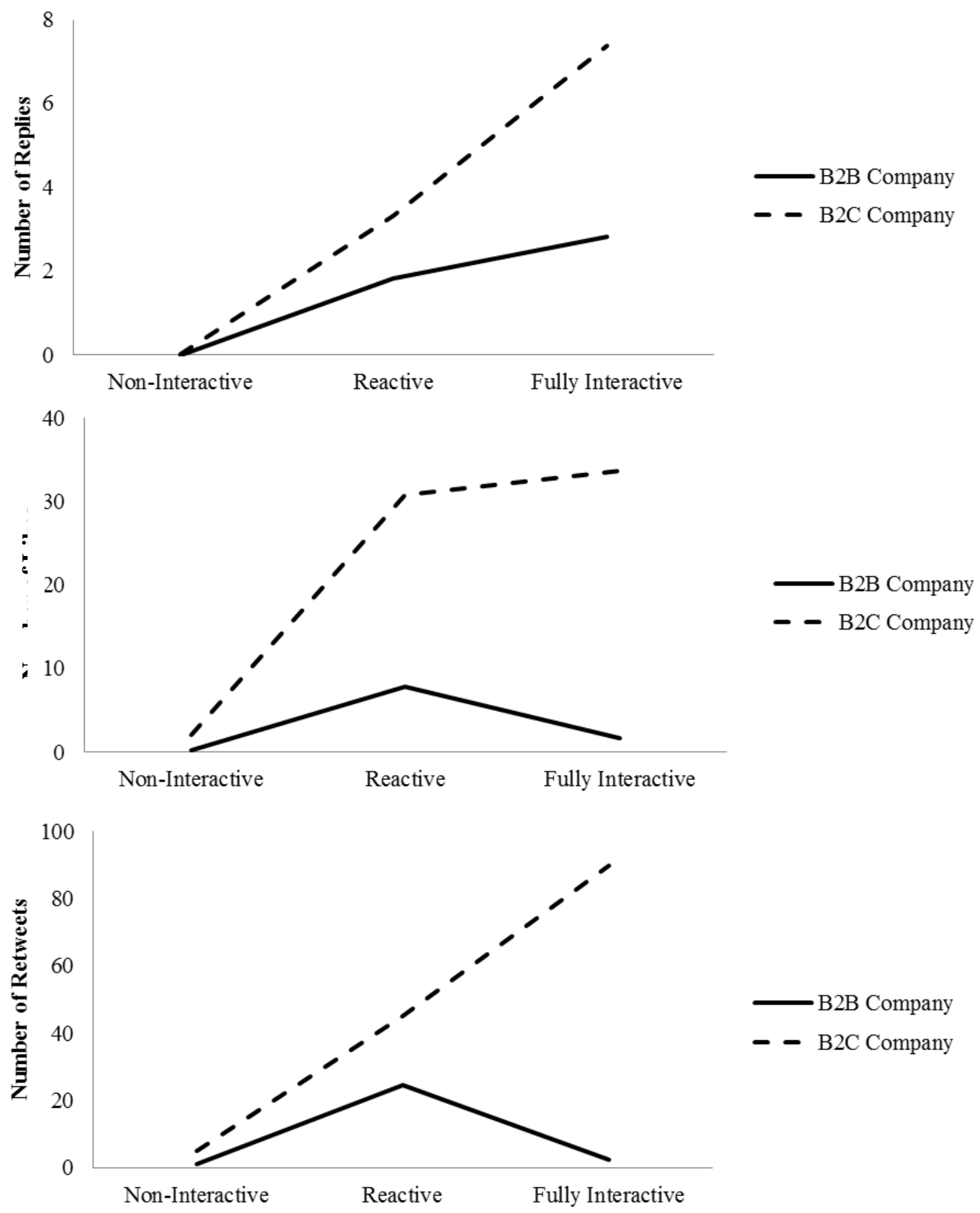

Fig. 1. Interaction between interactivity and company type on a) number of replies, b) number of retweets, and c) number of likes

$\mathrm{H} 2$ through $\mathrm{H} 4$ examine the influence of reply valence and company type on response time. A two-way ANOVA test showed a close-to-significant main effect of valence (three levels: positive, neutral, and negative) on response time $(F(2,150)=2.90, p=$ .058). Companies took less time to respond to positive $(M=574.63)$ and negative replies $(M=841.93)$ than neutral replies $(M=1,808.81)$. Thus, valence was further coded as two levels, neutral replies versus nonneutral replies. A 2 (neutral vs. non-neutral) $\times 2$ (B2B vs. B2C) ANOVA test was conducted. The results indicated significant main effects of company type
$(F(1,152)=6.86, p<.05)$ and reply valence $(F(1$, $152)=6.16, p<.05)$. Companies on average took 10.46 hours $\left(M_{\text {non-neutral }}=627.34\right)$ to respond to a positive or negative reply, and 30.15 hours $\left(M_{\text {neutral }}=\right.$ $1,808.82$ ) for a neutral reply. B2C companies took an average of 14.1 hours $\left(M_{B 2 C}=846.37\right)$ to respond to a user reply, which was significantly less than 55.01 hours for B2B companies $\left(M_{B 2 B}=3,300.30\right)$. According to these results, $\mathrm{H} 2$ was partially supported and $\mathrm{H} 3$ was fully supported. However, the interaction term was found to be nonsignificant, $F(1,152)=3.12$, $p=.079$. H4 was not supported. 


\subsection{Answer to research question}

To answer the proposed research question, a multilevel modeling analytical technique was used, taking both individual (micro-level) and organizational (macro-level) influences into consideration (Scheufele, Haas, \& Brosius, 2011). There were 311 units (companies) at the macrolevel in this analysis, making the investigation of cross-company comparisons possible (Raudenbush \& Bryk, 2002).

In the three multilevel models, the number of replies, retweets, and likes served as the outcome, respectively. Valence of user replies with two levels (non-negative vs. negative) was the key micro-level factor, which was included in the level-1 models. Another level-1 factor was interactivity, which had two levels (reactive vs. interactive). Company type, as a macro-level factor, was added into both intercepts and slopes of valence and interactivity in the level-2 model. The details of the analysis results are presented in Table 1. For B2B companies with a negative user reply, a tweet's likelihood to be retweeted decreased by $35.53 \%\left(\exp \left(\gamma_{10}\right)=.64\right.$, $\left.\gamma_{10}=-.44, p<.001\right)$, after controlling the interactivity level. For B2C companies with a negative user reply, the likelihood to be retweeted decreased by $23.11 \%\left(\exp \left(\gamma_{10}+\gamma_{11}\right)=.77, \gamma_{11}=\right.$ $.18, p<.05)$, after controlling the interactivity level. Thus, tweets from B2C companies were less impacted by negative user replies in terms of retweets. Similar results were discovered for number of likes. For both company types with a negative reply, a tweet would less likely be "liked" by users after controlling the level of interactivity. Similarly, this decrease in likelihood of being "liked" was more noticeable for B2Bs $(21.57 \%$, exp $\left.\left(\gamma_{10}\right)=.78, \gamma_{10}=-.24, p<.05\right)$ than for B2Cs $\left(3.65 \%, \exp \left(\gamma_{10}+\gamma_{11}\right)=.96, \gamma_{11}=.21, p<.05\right)$. The valence of user reply and company type had no significant impact on the likelihood of being replied to (all $p \mathrm{~s}>.05)$.

Table 1. Summary of multilevel modeling analysis results

\begin{tabular}{|c|c|c|c|c|c|c|}
\hline & \multicolumn{2}{|c|}{ Number of replies } & \multicolumn{2}{|c|}{ Number of retweets } & \multicolumn{2}{|c|}{ Number of likes } \\
\hline For intercept $\beta_{0}$ & coefficient & $p$ & coefficient & $p$ & coefficient & $p$ \\
\hline Intercept, $y_{00}$ & .469 & $<.001$ & .746 & $<.001$ & -.213 & .251 \\
\hline Company type, $y_{01}$ & .525 & $<.001$ & .670 & $<.001$ & .831 & $<.001$ \\
\hline \multicolumn{7}{|l|}{ For valence slope $\beta_{1}$} \\
\hline Intercept $2, \gamma_{10}$ & -.021 & .863 & -.439 & $<.001$ & -.243 & .016 \\
\hline Company type, $y_{11}$ & -.036 & .782 & .176 & .011 & .206 & .044 \\
\hline \multicolumn{7}{|c|}{ For interactivity slope $\beta_{2}$} \\
\hline Intercept $2, \gamma_{20}$ & .488 & .002 & -.084 & .597 & -.140 & .327 \\
\hline Company type, $\gamma_{21}$ & -.249 & .135 & 1.022 & $<.001$ & .568 & $<.001$ \\
\hline Random effects & Var. comp. & $p$ & Var. comp. & $p$ & Var. comp. & $p$ \\
\hline Intercept $1, u_{0}$ & .380 & $<.001$ & 2.493 & $<.001$ & 3.107 & $<.001$ \\
\hline
\end{tabular}

\section{Discussion}

This study examined the Fortune 500's Twitter use by focusing on interactivity, company type, and user reply valence. Contingency interactivity, or strategic communication based on reciprocity, was the focus. It is important to appear responsive from a company perspective because effective contingency interactivity leads to improved reputation and increased loyalty (Lee \& Park, 2013). In line with previous interactivity studies (Coyle \& Thorson, 2001; Song \& Zinkhan, 2008; Sundar et al., 2003), the results of this study supported and expanded Rafaeli's (1988) interactivity theory to the context of eWOM on Twitter. Interactivity can generate more engagement in the form of replies, retweets, and likes. Twitter can be many different things to different companies, but if the goal is to increase engagement, companies should interact often with individual users.
This study also tested how message valence affected engagement. Aligning with previous research, negativity does grab companies' attention as complaints did receive quick responses. It appears that companies only respond to user replies on the extremes of positivity and negativity, but neutral replies receive company responses when it is convenient. Looking only at neutral versus non-neutral replies, both $\mathrm{B} 2 \mathrm{Bs}$ and $\mathrm{B} 2 \mathrm{Cs}$ responded to non-neutral replies significantly faster, further strengthening the notion that extreme replies receive the quickest company responses.

Finally, this study confirmed that negative comments in general have a negative effect on eliciting users' engagement behaviors. Users place a good deal of emphasis on online negativity (Lee et al., 2009), which often leads to a decrease in future purchase decisions (Schultz et al., 2011). Though there are lab experiments demonstrating that negative comments under certain conditions could elicit favorable 
attitudes, this study revealed that negative user comments by and large have a dampening effect on company engagement in the form of retweets and likes for both B2Bs and B2Cs. This study used a multilevel modeling technique when examining interactivity, company type, and reply valence. Based on the results, $\mathrm{B} 2 \mathrm{Bs}$ suffer far more than $\mathrm{B} 2 \mathrm{Cs}$ when receiving a negative reply. B2Bs have a significantly higher chance of receiving fewer retweets and likes than B2Cs when receiving a negative user reply. Such differences can be interpreted from multiple angles. First, B2Cs have a significantly larger following than B2Bs on average so most activity concerning B2Bs on Twitter will generate fewer retweets and likes. Second, B2Bs tend to follow other B2Bs in a practice known as self-reflection (Campbell et al., 2010) and because these companies are concerned with relationship making (Lemke et al., 2011), retweeting or liking a negative reply directed at another company would be perceived as poor form. Lastly, B2Cs such as airlines or utility companies deal with negativity on a daily basis and in an example of schadenfreude, users may be more willing to retweet or like a tweet that received negative replies just to rub it in $\mathrm{B} 2 \mathrm{C}$ companies' faces, so to speak. Because negative replies directed toward B2Cs can draw more attention to the negative situation than with B2Bs, B2Cs need to address users' concerns faster than B2Bs to reduce the chances of other users viewing the replies, retweets, and likes. Truthfully, B2Cs and B2Bs should both address negative replies as quickly as possible, but because a B2C's audience is so much larger than a B2B's, it is imperative for a B2C to act the quickest.

\section{Conclusion}

The results from this study contribute theoretically and practically to the discipline of strategic communication. From a theoretical perspective, public relations is simply the management of credibility (Stacks, 2017). To effectively manage credibility in today's social media world, companies should tweet often and respond to user replies as quickly as possible to create a culture of interactivity. Doing this aligns with the International Public Relations Association's definition of public relations which is to reach a harmony through mutual understanding based upon truth and full information. The harmony, in this case, is increased user engagement as this study revealed that interactive tweets generate more replies, retweets, and likes than one-way, top-down traditional mass media communication.

From a practical perspective, companies utilizing Twitter should incorporate the following strategies based on the results of this study:
Tweet for interactivity: Companies should strive for interactivity when tweeting. Forcing traditional, topdown strategies into Twitter will not generate engagement. Instead, companies should ask questions, hold contests, entertain, inform, and address the needs of users. Users are actively searching for value online and within the realm of social media. The companies that can provide valuable tweets will in turn receive user interaction that generates engagement.

Address extreme replies quickly: Positive and negative replies received the fastest responses from companies in this study. The neutral replies received the slowest responses. Users expect their replies to receive a response on Twitter, but it is unrealistic to expect a company to address every user reply. Instead, companies should thank users sending positive replies and address the concerns from users sending negative replies. This demonstrates a culture of listening. Responses should come quickly as well because fast responses convey sincerity on Twitter.

Understand your business: Users expect a social presence in today's digital environment. A B2C should absolutely be on Twitter because its customers use Twitter. The B2C should interact and do so quickly because that is the expectation from users. It is a little more complicated for a B2B. B2Bs do business with other companies but companies are comprised of people and people use social media. However, B2Bs generate less engagement overall and receive a more potent dampening effect when a user sends a negative reply. For these reasons, the temptation for B2Bs is to avoid social media altogether. However, the wise decision is for a B2B to use Twitter and to benchmark itself against other B2Bs and competitors within the industry. In short, B2Cs should be compared to other B2Cs and B2Bs should be compared to other B2Bs. Understand your business, target audience, and competitors, and your Twitter strategy will be genuine leading to increased credibility perceptions from users.

This study revealed that when the Fortune 500 displayed full interaction, engagement in the form of replies, retweets, and likes increased. Companies need to respond faster to users on Twitter and realize that negative replies have the potential to decrease engagement. While negative replies are perhaps unavoidable to an extent, companies should operate in such a way to give users no reason to tweet with a negative tone. Positive business operations are more necessary than ever now that every user has a potentially prominent voice on Twitter. Because social media issues have a way of metastasizing into a viral crisis, B2Cs and B2Bs should form a social media team armed with a detailed plan to address negative replies as strategically and quickly as possible. 


\section{References}

1. Bach, S., \& Kim, S. (2012). Online consumer complaint behaviors: The dynamics of service failures, consumers' word of mouth, and organization-consumer relationships. International Journal of Strategic Communication, 6(1), 59-76. https://doi.org/10.1080/1553118X.2011.634871

2. Berthon, P., Ewing, M., Pitt, L., \& Naudé, P. (2003). Understanding B2B and the Web: The acceleration of coordination and motivation. Industrial Marketing Management, 32(7), 553-561. https://doi.org/10.1016/S00198501(02)00279-1

3. Campbell, C., Papania, L., Parent, M., \& Cyr, D. (2010). An exploratory study into brand alignment in B2B $\begin{array}{lllll}\text { relationships. } & \text { Industrial Marketing } & \text { Management, } & 39(5), 720 .\end{array}$ https://doi.org/10.1016/j.indmarman.2010.02.009

4. Chen, Z., \& Lurie, N. (2013). Temporal contiguity and negativity bias in the impact of online word of mouth. Journal of Marketing Research, 50(4), 463-476. https://doi.org/10.1509/jmr.12.0063

5. Eisend, M. (2013). The Moderating influence of involvement on two-sided advertising effects. Psychology \& Marketing, 30(7), 566-575. https://doi.org/10.1002/mar.20628

6. Etter, M. (2013). Reasons for low levels of interactivity: (Non-) interactive CSR communication in twitter. Public Relations Review, 39(5), 606-608. https://doi.org/10.1016/j.pubrev.2013.06.003

7. Himelboim, I., McCreery, S., \& Smith, M. (2013). Birds of a feather tweet together: Integrating network and content analyses to examine cross-ideology exposure on Twitter. Journal of Computer-Mediated Communication, 18(2), 154-174. https://doi.org/10.1111/jcc4.12001

8. Kalman, Y., \& Rafaeli, S. (2011). Online pauses and silence: Chronemic expectancy violations in written computer-mediated communication. Communication Research, $\quad 38(1)$, 54-69. https://doi.org/10.1177/0093650210378229

9. Kalman, Y., Ravid, G., Raban, D., \& Rafaeli, S. (2006). Pauses and response latencies: A chronemic analysis of asynchronous CMC. Journal of Computer-Mediated Communication, 12(1), 1-23. https://doi.org/10.1111/j.10836101.2006.00312.x

10. Karimova, G. (2011). Can level of interactivity be measured? Empedocles: European Journal for the Philosophy of Communication, 2(2), 291-304. https://doi.org/10.1386/ejpc.2.2.291_1

11. Kiousis, S. (2002). Interactivity: A concept explication. New Media \& Society, 4(3), 355-383. https://doi.org/10.1177/146144480200400303

12. Lee, H., \& Park, H. (2013). Testing the impact of message interactivity on relationship management and organizational reputation. Journal of Public Relations Research, 25(2), $188-206$. https://doi.org/10.1080/1062726X.2013.739103

13. Lee, M., Rodgers, S., \& Kim, M. (2009). Effects of valence and extremity of eWOM on attitude toward the brand and website. Journal of Current Issues \& Research in Advertising, 31(2), 1-11. https://doi.org/10.1080/10641734.2009.10505262

14. Lee, M., \& Youn, S. (2009). Electronic word of mouth (eWOM): How eWOM platforms influence consumer product judgement. International Journal of Advertising, 28(3), 473-499. https://doi.org/10.2501/S0265048709200709

15. Lohtia, R., Donthu, N., \& Hershberger, E. (2003). The impact of content and design elements on banner advertising click-through rates. Journal of Advertising Research, 43(4), $410-418$. https://doi.org/10.1017/S0021849903030459

16. Martin, P. (2008). The mass media as sentinel: Why bad news about issues is good news for participation. Political Communication, 25(2), 180-193. https://doi.org/10.1080/10584600801985706

17. Newhagen, J. E., Corders, J. W., \& Levy, M. R. (1995). Nightly@nbc.com: Audience scope and the perception of interactivity in viewer mail on the Internet. Journal of Communication, 45(3), 164-175. https://doi.org/10.1111/j.1460-2466.1995.tb00748.x

18. Parmar, B. (2015). 50 Companies That Get Twitter - and 50 That Don't. Retrieved from https://hbr.org/2015/04/the-best-and-worst-corporate-tweeters

19. Rafaeli, S. (1988). Interactivity: From new media to communication. In R. Hawkins, J. Weimann, \& S. Pingree (Eds.), Advancing communication science: Merging mass and interpersonal processes (pp. 110-134). Newbury Park, CA: Sage.

20. Raudenbush, S. W., \& Bryk, A. S. (2002). Hierarchical linear models: Applications and data analysis methods (2nd ed.).Thousand Oaks, CA: Sage.

21. Rapp, A., Beitelspacher, L., Grewal, D., \& Hughes, D. (2013). Understanding social media effects across seller, retailer, and consumer interactions. Journal of the Academy of Marketing Science, 41(5), 547-566. https://doi.org/10.1007/s11747-013-0326-9

22. Reber, B., \& Fosdick, S. (2005). Building business relationships online: Relationship management in business-tobusiness e-commerce. Journal of Website Promotion, 1(1), 13-29. https://doi.org/10.1300/J238v01n01_03

23. Rim, H., \& Song, D. (2016). "How negative becomes less negative": Understanding the effects of comment valence and response sidedness in social media. Journal of Communication. Retrieved from http://onlinelibrary.wiley.com/doi/10.1111/jcom.12205/abstract 
24. Roering, K., \& Paul, R. (1976). The effect of the consistency of product claims on the credibility of persuasive messages. Journal of Advertising, 5(2), 32-36. https://doi.org/10.1080/00913367.1976.10672634

25. Saffer, A., Sommerfeldt, E., \& Taylor, M. (2013). The effects of organizational Twitter interactivity on organization-public relationships. Public Relations Review, 39(3), 213-215. https://doi.org/10.1016/j.pubrev.2013.02.005

26. Schandorf, M. (2013). Mediated gesture: Paralinguistic communication and phatic text. Convergence: The International Journal of Research into New Media Technologies, 19(3), 319-344. https://doi.org/10.1177/1354856512439501

27. Scheufele, B., Haas, A., \& Brosius, H. (2011). Mirror or molder? A study of media coverage, stock prices, and trading volumes in Germany. Journal of Communication, 61(1), 48-70. https://doi.org/10.1111/j.14602466.2010.01526.x

28. Schultz, F., Utz, S., \& Göritz, A. (2011). Is the medium the message? Perceptions of and reactions to crisis communication via Twitter, blogs and traditional media. Public Relations Review, 37(1), 20-27. https://doi.org/10.1016/j.pubrev.2010.12.001

29. Song, J., \& Zinkhan, G. (2008). Determinants of perceived web site interactivity. Journal of Marketing, 72(2), 99113. https://doi.org/10.1509/jmkg.72.2.99

30. Stacks, D. W. (2017). Primer for public relations research. New York: Guilford Press.

31. Steyn, P., Salehi-Sangari, E., Pitt, L., Parent, M., \& Berthon, P. (2010). The social media release as a public relations tool: Intentions to use among B2B bloggers. Public Relations Review, 36, 87-89. https://doi.org/10.1016/j.pubrev.2009.09.005

32. Stromer-Galley, J. (2004). Interactivity-as-product and interactivity-as-process. The Information Society, 20(5), 391-394. https://doi.org/10.1080/01972240490508081

33. Steuer, J. (1992). Defining virtual reality: Dimensions determining telepresence. Journal of Communication, 42(4), 73-93. https://doi.org/10.1111/j.1460-2466.1992.tb00812.x

34. Sundar, S. (2004). Theorizing interactivity's effects. The Information Society, 20(5), 385-389. https://doi.org/10.1080/01972240490508072

35. Sundar, S. S., Kalyanaraman, S., \& Brown, J. (2003). Explicating web site interactivity: Impression formation effects in political campaign sites. Communication Research, 30(1), 30-59. https://doi.org/10.1177/0093650202239025

36. Sweetser, K. (2010). A losing strategy: The impact of nondisclosure in social media on relationships. Journal of Public Relations Research, 22(3), 288-312. https://doi.org/10.1080/10627261003614401

37. Usunier, J., \& Roulin, N. (2010). The influence of high- and low-context communication styles on the design, content, and language of business-to-business web sites. Journal of Business Communication, 47(2), $189-227$. https://doi.org/10.1177/0021943610364526

38. Waters, R., \& Williams, J. (2011). Squawking, tweeting, cooing, and hooting: Analyzing the communication patterns of government agencies on Twitter. Journal of Public Affairs, 11(4), 353-363. https://doi.org/10.1002/pa.385

39. Westerman, D., Spence, P., \& Heide, B. (2014). Social media as information source: Recency of updates and credibility of information. Journal of Computer-Mediated Communication, 19(2), 171-183. https://doi.org/10.1111/jcc4.12041

40. Wise, K., Hamman, B., \& Thorson, K. (2006). Moderation, response rate, and message interactivity: Features of online communities and their effects on intent to participate. Journal of Computer-Mediated Communication, 12(1), 24-41. https://doi.org/10.1111/j.1083-6101.2006.00313.x

41. Wu, G. (1999). Perceived interactivity and attitude toward websites. In M. S. Robert (Ed.), Proceedings of the American Academy of Adverting (pp. 254-262). Gainesville, FL. 